\title{
MCRP: multiple chain routing protocol for energy efficiency in homogeneous wireless sensor networks
}

\begin{abstract}
Wireless sensor networks (WSNs) comprise a wide variety of applications. In most of the applications, the sensor nodes are distributed in a hostile area and nodes in WSNs are energy constrained, once it is installed, it would be difficult to recharge the energy source of those sensor nodes. There are many solutions that can be used to overcome the energy limitation issue and one of the main solutions is the routing algorithm used in the network. Routing algorithms in WSNs are responsible for maintaining and discovering the suitable routes in the networks. Therefore, this research presents a Multiple Chain Routing Protocol for Energy Efficiency in Homogeneous Wireless Sensor Networks (MCRP). The major objectives of the MCRP protocol are to increase the lifetime of WSNs by reducing the data transmission path to minimize energy consumption in WSN, and to maximize the network stability period by distributing the load evenly among all nodes. The MCRP works within two stages: the initialization stage and the data transmission stage. Simulation results demonstrate that the presented algorithm MCRP achieves its design goals and outperforms the existing work such as Chain-Chain based routing protocol (CCBRP), and Two-stage chain routing protocol (TSCP) algorithms in terms of Network lifetime, FND and LND (first node and last node died), Network stability period, and Energy consumption.
\end{abstract}

Keyword: Routing algorithms; Wireless sensor networks; Network lifetime; Energy consumption; Load management 